\title{
A SURVEY FOR DATA MINING FRAME WORK FOR POLYMER MATRIX COMPOSITE ENGINEERING MATERIALS DESIGN APPLICATIONS
}

\author{
DORESWAMY \\ Department of Post-Graduate Studies and Research in Computer Science, \\ Mangalore University, \\ Mangalagangotri-574 199, \\ Karnataka, INDIA, \\ Ph.No:+91-824-2287670 \\ Email: doreswamyh@yahoo.com \\ http://www.mangaloreuniversity.ac.in
}

Received: 11-04-2008

Revised: $13-11-2008$

\begin{abstract}
In this paper, a survey for Data Mining frame work has been done for proposing Data Mining methodologies to engineering materials design applications. An exhaustive literature survey made in this article has covered the modeling systems such as Analytical Model, Numerical Simulation Model and Computer Based Modeling Systems, which were developed and implemented for Polymer Composite processing from the year 1950 to till 2006. Motivation for the present investigation is inspired by the Computer Based Models and is depicted as Mining Frame Work for determining optimal decision making strategies and performing intelligent computational operations associated to advanced Composite materials design applications. Data Mining and Knowledge Discovery has made tremendous progress in Computer Science in the last 15 years. However, a large gap exists between the results of Data Mining and Knowledge Base system that can provide and support proper decision making. Though many Modeling and Simulation Systems have been designed and developed for improving the concurrent engineering materials design throughput, the application of Data Mining is still essential to extract previously unknown and potentially useful information and knowledge from the engineering materials database. The information and knowledge extracted by the Mining System is enormously useful for Composite materials design applications and for reducing the materials selection cost and time that is required to select the suitable constituent materials that maximize the performance of Composite materials under deferent environmental conditions.
\end{abstract}

Keywords: Data Mining and Knowledge Discovery, Composite Materials Selection, Machine Learning, Neural Network Model.

\section{Introduction}

Many of the modern technologies require composite materials with unusual combinations of properties that can not be met by the conventional metal alloys, ceramic and polymeric materials properties [72]. This is especially true for materials that are required for aerospace, underwater, and transportation applications. Composite materials are the materials that consist of more than one class of materials and whose properties are better than its constituent materials. It consists of two phases: one is called Matrix, which is continuous and surrounds the other phase, which is often called as reinforced or dispersed phase. A composite material may be designed by more than one class materials such as polymer, ceramic and metal. A combination of a class of Polymer/Ceramic/Metal material with reinforced fiber 
material yields, respectively, Polymer Matrix Composite (PMC), Ceramic Matrix Composite (CMC) and Metal Matrix Composite (MMC). Since polymeric materials are lightweight, having high stiffness and replaces conventional materials in many applications, a brief description of polymer and polymer matrix composite materials and their benefits are illustrated below.

\subsection{Polymers}

Polymers are a large class of materials consisting of many small molecules called monomers that can be linked together to form long repetitive chains. A typical polymer can include tens of thousands of monomers. A model of a very simplistic polymer is -A-A-A-A-A-A-Awhere " $A$ " is the monomer and each " $A$ " is linked to another " $A$ " making it many units together, a polymer[1].

Man has taken advantage of the versatility of polymers for centuries in the form of oils, tars, resins, and gums. However, it was not until the industrial revolution that the modern polymer industry began to develop. In the late 1830s, Charles Goodyear succeeded in producing a useful form of natural rubber through a process known as "vulcanization [1]. " Some 40 years later, Celluloid (a hard plastic formed from nitrocellulose) was successfully commercialized. Despite these advances, progress in polymer science and technology was slow until the 1930s [2], when materials such as Vinyl, Neoprene, Polystyrene, and Nylon were developed. The introduction of these revolutionary materials began an explosion in polymer research that is still going on today.

Unmatched in the diversity of their properties, polymers such as Cotton, Wool, Rubber, Teflon(tm), and all plastics are used in nearly every industry. Synthetic or engineering polymers and their sub types thermosets and thermoplastics could be produced with a wide range of stiffness, strength, heat resistance, density, and even at low price. With continued research into the Material Science and Technology applications, the polymers and polymer matrix composites play an ever increasing role in modern society.

\subsection{Polymer Matrix Composites}

Polymer Matrix Composites are lightweight, strong, and energy-efficient materials that offer significant advantages to durable-goods manufacturers and to performance- driven markets such as the aerospace industry [3][4]. Polymer composites consist of a reinforcing structural constituent and a protective polymer matrix. The properties of the combined material are significantly better than the sum of the properties of each component, giving materials with high strength-to-weight ratios. As a result, polymer composite parts are generally 20 to $30 \%$ lighter than the corresponding metal parts.

Polymer Matrix Composite (PMC), also called fiber reinforced polymer composite(FRPC), is defined as a polymer (plastic) matrix, either thermosets or thermoplastic, that is reinforced (combined) with a fiber or other reinforcing material with a sufficient aspect ratio (length to thickness) to provide a discernable reinforcing function in one or more directions. PMCs are different from traditional construction materials such as steel or aluminum. PMCs are anisotropic (properties only apparent in the direction of the applied load) whereas steel or aluminum is isotropic (uniform properties in all directions, independent of applied load). Therefore, PMCs properties are directional, meaning that the best mechanical properties are in the direction of the fiber placement. Composites are similar to reinforced concrete where the fiber is embedded in an isotropic matrix called concrete.

\subsection{Composition}

Composites are composed of resins, reinforcements, fillers or/and additives. Each of these constituent materials or ingredients plays an important role in the processing and final performance of the end product. The resin or polymer is the "glue" that holds the composites together and influences the physical properties of the end product. The reinforcement provides the mechanical strength. The fillers and additives are used as process or performance aids to impart special properties to the end product. The mechanical properties and composition of PMCs can be tailored for their intended use. The type and quantity of materials selected in addition to the manufacturing process to fabricate the product will affect the mechanical properties and performance. Important considerations for the design of composite products include type of fiber reinforcement, percentage of fiber or fiber volume, orientation of fiber $\left(0^{\circ}, 90^{\circ},+/-45^{\circ}\right.$ or a combination of these), type of resin , cost of product, volume of production (to help determine the best 
manufacturing method), manufacturing process and service conditions.

\subsection{Benefits}

PMCs have many benefits in their selection and use in modern life. The selection of the materials depends on the performance and intended use of the product. The composite designer can tailor the performance of the end product with proper selection of materials. It is important for the end-user to understand the application environment, load performance and durability requirements of the product and convey this information to the composite industry professionals. A summary of composite material benefits include: light weight, high strength-to-weight ratio, directional strength, corrosion resistance, weather resistance, dimensional stability, low thermal conductivity, low coefficient of thermal expansion, radar transparency, non-magnetic, high impact strength, high dielectric strength (insulator), low maintenance, long term durability, part consolidation, small to large part geometry possible and tailored surface finish

The rest of this paper has been organized as follows: The second section emphasizes the detailed literature survey on models and expert systems designed and developed on polymer matrix composite materials design and its applications. The third section describes the scope of data mining frame work for materials design applications. The forth section describes the proposed Data Mining frame work models and the fifth section depicts the data evaluation and representation and the sixth section gives the conclusion and future scope of research.-

\section{Literature Survey}

\subsection{Earlier Work}

The first serious efforts on modeling of polymer processing operations were carried out at DuPont, Delaware, U.S.A., and subsequently published in the early fifties. Also, Maillefer in Switzerland developed, independently from the DuPont team, some very important models for polymer extrusion at about the same time. The contributions of McKelvey, Gore and Squires of DuPont are well known. Bernhardt's book [3] summarized just about everything important on polymer process modeling until about 1958. McKelvey's book [4] was perhaps the first ever and very successful attempt to present a unified approach in the framework of the equations of conservation of mass, momentum and energy and the change of phase mechanisms. Klein and Marshall's book [5] was perhaps the first monograph ever exclusively devoted to computer modeling of polymer processing but it had very little impact, because the material was really outdated in the seventies. Tadmor and Klein's book [6] presented the first complete model for plasticating extrusion including transport of solids from the hopper forward, as the screw rotates, melting and melt pumping. Package for plasticating extrusion called EXTRUD [7], which was based on the models described in Tadmor and Klein's book [4], became commercially available in the early seventies.

In the seventies many investigators in universities and industry worked on various computer models for simulating extrusion [7], filling process [8][9], calendaring [18] and other polymer processes[25-34]. However, there was little impact of the computer models on process technology till 1978 when C. Austin produced the first MOLDFLOW package [8] for injection mold filling. In the early eighties the art of mold design started to become an engineering discipline heavily relying on computer predictions with the release of C-MOLD [9] and other software packages exclusively devoted to the injection molding process [10].

In the eighties also, many rigorous investigations on various aspects of polymer flows through channels, dies and process equipment were carried out by various research groups in North America and Europe. Computer simulation packages for polymer flows such as FIDAP [11], POLYFLOW [12], NEKTON [13] and POLYCAD ${ }^{\circledR}[14]$ became commercially available.

In the nineties there was more emphasis on processspecific application of computer methods for such processes as twin-screw extrusion [24-26], thermoforming, compression molding [9], film blowing [28], reaction injection molding [15], and gas-assisted injection molding [15]. The greatest technological impact of computer models was in injection molding. The reason is the ability of the Hele-Shaw flow approximation [15] to describe reasonably well the mold filling process. The 
commercially available packages [11-14] can handle the majority of problems for 1-D, 2-D and 3-D flows. The determination of free surfaces or interfaces is the subject of current research for 3-D flows. Karagiannis et al [16][17] have addressed some research issues relating to 3-D flow computer simulation.

The computer modeling and simulation packages [1114] [24-34] developed for polymer processing were certainly not limited to numerical analysis and graphical visualizations. The incorporation of Expert System and Knowledge Bases into modeling process, especially in interpretations of results obtained from modeling techniques [11-14][19-34] for polymer composite processing lead to a new and exiting idea called the Knowledge-Based System(KBS) or Expert Decision Making System to find solutions to many applications for which the traditional computer modeling systems do not lend optimal solutions. Knowledge based and Artificial Intelligence techniques were proposed as powerful tools for modeling applications in computer aided polymer processing analysis and design [35][36].

Over the last two decades, knowledge-based techniques [37][40][44][53-54] emerged as powerful decision support tools for modeling polymer composite process. More emphasis and efforts were made by the scientists working in Intelligent Systems Laboratory (ISL) at Michigan State University, U.S.A., towards developing Intelligent Decision Support Systems [41-50] that aid the solution of complex problems through precompiled domain knowledge and specific inferencing techniques. ISL developed domain based Intelligent Decision Support Systems such as COMADE [51] for specifying the combinations of polymer matrix materials, chemical agents (curing, reactive diluents), fiber materials and fiber lengths. The design of polymer composite material systems specifies nothing other than determining valid combinations of material system constituents. COMADE provided a focus for composite materials system design and also presented possibilities for families of composite material systems that may not be immediately obvious. It considered the performance requirements (e.g., tensile strength, flexural modulus) and the environmental conditions (e.g., chemical environment, use temperature), an assembly may face and generate multiple material system designs. COMADE can generate over one thousand material system designs, ranging from simple polyesters to exotic thermoplastic systems. COMADE does not consider fabrication issues, as that portion of the composites' design process is handled in a separate system.

COFATE [52] is the current system at ISL for the selection of polymer composite fabrication technologies such as filament winding, injection molding, lay-up, calendaring etc. Each of the fabricating processes has its own specific processing concerns and limitations. The expanse of knowledge required to consider even a fraction of the options available for processing a part is quite substantial. The selection of a fabrication process for a polymer composite assembly affords a prime opportunity to use intelligent decision support systems. The selection of a process in polymer composites is extremely knowledge intensive due to the myriad concerns within each of the various fabrication technologies.

Besides the intelligent decision support systems developed at ISL, researchers have developed generating tools such as Part Designer (CPD) [53], the Composite Designer (COMDES) [54], and Expert Assisted Design of Composite Structures (EADOCS) [55]. These developed tools could share a conceptual design philosophy of expert systems designed by ISL. ISL has taken further knowledge-based steps to integrate the developed expert systems COMADE [51], COFATE [52] and other supporting tools [53-55] into a single polymer composite processing system for transforming the intellectual ideas on materials and their properties and the enhanced functional capabilities of polymer processing systems for advanced polymer matrix composite designs.

The technical report [56] submitted to the 1993 American Society of Mechanical Engineers and cosponsored by the IGTI and ASME, Cincinnati, emphasized the potentials of fuzzy sets and neural networks under soft computing framework[57] for aiding in all aspects of manufacturing of advanced materials like metals, ceramics and polymers. This report briefly introduced the concepts of fuzzy sets and neural networks and showed how they could be used in the design of advanced materials and manufacturing processes. These two computational methods are alternatives to other methods such as the Taguchi method [14].

In spite of several research attempts to design computer based expert systems for polymers and their 
composite processing [1-56], there is no concise integrated expert systems for the systemic analysis and design of advanced composite materials. The detailed investigation on intelligence decision support systems has led to Data Mining frame work for advanced composite materials design and analysis. This has been depicted as challenging interdisciplinary research[58-73] in Data Mining and Knowledge Discovery Process from materials databases, which is a part and partial filed of Computer Science and Technology and for merging triangular socio-economic-technical bridges between the Material Science and Technology (MST) and the Computer Science and Information Technology (CS\&IT) fields.

\section{Data Mining Frame Work}

With recent developments in data transfer and network technologies, data collection technologies in many emerging scientific applications need for a paradigm shift from a traditional hypothesize-and test process to a partial automation of hypothesis generation, model construction and experimentation. To develop appropriate knowledge discovery models associated to advanced composite materials technologies such as fabrication technologies, Nano technology and composite materials curing technologies, various data mining models to be designed and integrated to automate the mining process and to obtain potentially useful and ultimately understandable patterns in domain database. Data Mining or Knowledge Discovery is a young sub-discipline of computer science aiming at the automatic interpretation of large data sets. The classic definition of knowledge discovery is "the non-trivial process of identifying valid, novel, potentially useful and ultimately understandable patterns in data" [73]. Data Mining or Knowledge Discovery in Databases (KDD) is a multidisciplinary area that integrates techniques from several fields including machine learning, statistics and database technology for analysis of large volume of data.

Knowledge discovery process is an interactive and iterative procedure involving the following basic steps [73].

1. Understanding the domain knowledge: Understating of application domain and prior knowledge about the problem helps go ahead for relevant pattern in the data set.

2. Data Preprocessing: It is a prerequisite operation to remove the unwanted data elements that decline the performance of the mining algorithms [71][ 73].

3. Data Collection and Integration: Data is gathered from different sources and is required to integrate as a data repository with common data retrieval format to search for the target data.

4. Data reduction and projection: It reduces the size of the data set to represent useful patterns to represent the data and transformed into another form to improve the performance of the mining algorithms.

5. Data Mining: It is the core task of knowledge discovery process. Mining algorithm from different fields may model to extract trivial and useful patterns. The classes of Data Mining techniques are association rule analysis, Classification and prediction, Cluster analysis, Soft computing approaches and statistical, machine learning and artificial intelligence methods.

6. Pattern Evaluation: It identifies truly interesting patterns representing knowledge based on interestingness measures.

7. Knowledgebase: This is the domain knowledge that is used to guide the search or evaluate interestingness measure of resulting patterns. Such knowledge can include concept hierarchy, knowledge such as user beliefs, which can be used to access pattern's interestingness based on its unexpectedness.

8. Data Visualization: Knowledge representation techniques are focused to represent the mined knowledge to the end user

\subsection{Proposed Data Mining Frame Work}

A typical data mining framework proposed for composite materials performance analysis is shown in figure 1 . It employs all the basic steps of data mining and Knowledge discovery process with data mining algorithms from statistical, machine learning and artificial intelligence classes. This accepts end user requirements from the graphical user interfaces and performs the following operations: 
Doreswamy

1. It classifies the input user requirements, usually properties of materials, $\left\{p_{1}, p_{2}, p_{3}, p_{4}, \ldots p_{n}\right\}$ by assigning class label to each property, $p_{i} \in C_{j}$. Then predicts the materials from the database that matches the maximum distributed properties in a class $C_{j=1 . . m}$ of the material class into which it belongs and then predicts a material that matches the user's input requirements.

2. It predicts a reinforcement fiber that maximizes composite performance, from the large fiber class, based on critical length of a fiber derived from matrix material property.

3. It predicts the cost-effective polymer matrix and reinforcement fiber that maximize the composite materials performance and reduce the cost of materials selection.

4. The mechanical performance of a composite material in which varying volume fraction and diameter of fibers uniformly placed at different orientations at different layers, is predicted to guide the composite design engineers in optimizing design strategies.

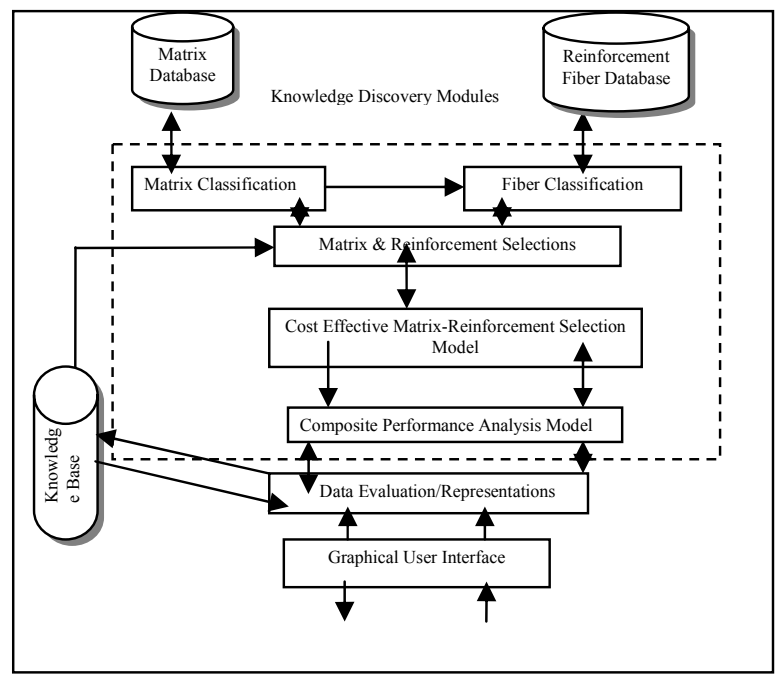

Figure 1: Data Mining Systems Architecture

\section{4 .Data Mining Models}

The proposed data mining system architecture has integrated with matrix and reinforced materials' databases, materials classification and selection Model, and Composite stiffness analysis model. Detail description of each model is given in subsequent sections.

\subsection{Materials Data Representation Model}

An object-oriented data model is proposed to organize materials data sets using objects, classes, sub classes, class hierarchy, data encapsulation, data binding and other advanced features as specialization and generalization, aggregation, summarization and unions. An objectoriented data model is a logical organization of the materials data sets as objects (entities), constraints and the cardinality relationships among objects. These objects are identified by unique ID is called object identifier. Similar objects are grouped together to form as a class. Every object has a state (the set of values for the attributes of the object) and a behavior (the set of methods - program code - which operate on the state of the object). The state and behavior encapsulated in an object are accessed or invoked from outside the object only through explicit message passing. Materials characterization and discrimination tasks are performed on the Materials database. Materials are classified into Polymer, Ceramic and Metal classes and the fibers may be classified into short, medium and long fiber based on the properties of both matrix and reinforcement fibers. A typical organization of the materials data set is shown below.

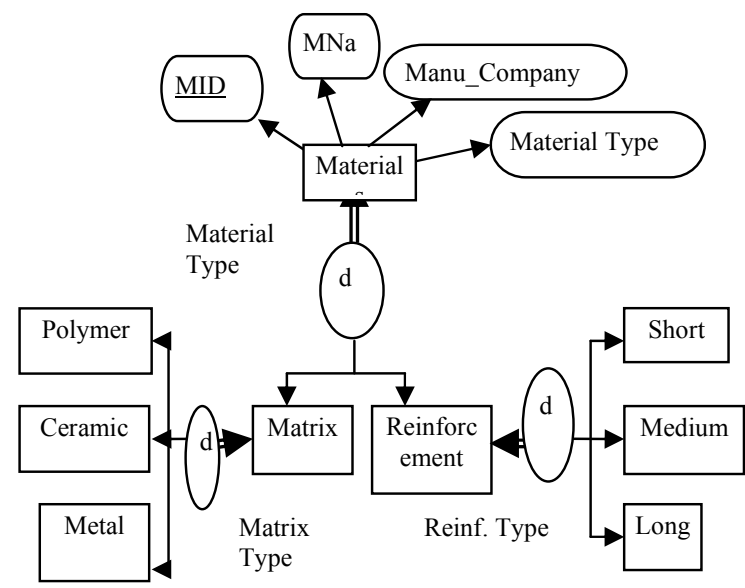

Figure 2: Cardinality and Disjoint Constraints in specialization hierarchy

\subsection{Matrix Materials Classification Model}


Classification is a data mining task for assigning a class label to a randomly selected data set from the materials data set. Back Propagation Neural Network model is proposed for classifying engineering materials into Polymer, Ceramic and Metal classes as it is performs well on on-linear data sets by minimizing classification error rate[76][77].

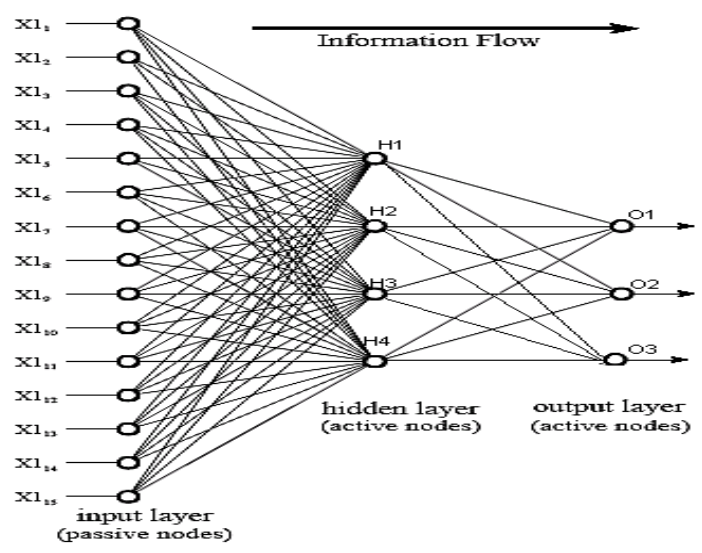

Figure 3: Typical MLPNN Architecture

The neural network model is a three layer feed forward neural network [57][58]. Each layer is fully connected to all successive layers through the connection weights as shown in figure 3. For a neuron $i$, the normalized weighted inputs are fed and then summed up to the final input, $u_{i}$

$u_{i}=\sum_{j=1}^{m} w_{j, i} x_{j}$

The inputs for neurons are propagated to outputs through the neurons in the hidden layers according to the following sigmoid activation function with bias $\theta$.

$f\left(u_{i}\right)=\frac{1}{1+e^{-\left(u_{i}+\theta\right)}}$

where $u_{i}$ is the input function and $f\left(u_{i}\right)$ is the output function.

The training procedure is a search algorithm to minimize the error between the input and the output patterns by changing the weights. This process determines the weights of $\mathrm{NN}$ connections to map the relationships between input and output. The network must be trained with training data sets in such a way that for a given input vector, the output vector must be obtained to classify the patterns.

When the back propagation learning method is used as training procedure, the objective function for an input/output pattern is the sum of the squared residual errors as follows.

$$
E=\frac{1}{2} \sum_{k=1}^{m}\left(T_{k}-O_{k}\right)^{2}
$$

where $T_{k}$ and $O_{k}$ are the target and the actual computed outputs of $k^{\text {th }}$ output unit respectively. To find a set of weights that minimizes the objective function, a gradient decent method is implemented. The weight change is proportional to the derivative of the error with respect to each weight. This can be expressed as

$$
\Delta w \propto \frac{\partial E}{\partial W}
$$

The determination of weight change is a recursive process which starts with the output units. For a weight that is connected to a unit in the output layer, its change is based on the error of this output unit. It is given by

$$
\begin{aligned}
& \Delta w_{k, j} \propto O_{k}\left(1-O_{k}\right)\left(T_{k}-O_{k}\right) O_{j}=\delta_{k} O_{j} \\
& \Delta w_{k, j}=\delta_{k} O_{j}
\end{aligned}
$$

where $\delta_{k}$ is referred to as the error signal at the $\mathrm{k}^{\text {th }}$ output unit. The output signals are back propagated to units in the hidden layer. The change of a weight in hidden layer is determined by

$\Delta w_{j, i} \propto O_{j}\left(1-O_{j}\right) \sum_{k} \delta_{k} w_{k, j} O_{i}$,

$\Delta w_{j, i}=\delta_{j} O_{i}$

In order to increase the speed of the training procedure without any oscillations, the adaptive learning rate and momentum are used during the training process. The equations (7) and (8) are then rewritten as follows

$\Delta w_{k, j}(n)=\eta \delta_{k} O_{j}+\alpha \Delta w_{k, j}(n-1)$

$\Delta w_{j, i}(n)=\eta \delta_{j} O_{i}+\alpha \Delta w_{j, i}(n-1)$

where $\mathrm{n}$ is the training epoch number, $\eta$ is the learning rate and $\alpha$ is the momentum. The momentum allows the previous weight change to have a continuing influence on the current weight change. 
Doreswamy

\subsection{Decision Tree Classifier for Fiber Classification}

It is a divide and conquer approach to the problem of learning from a set of independent test entities. The decision tree induction [80] method is implemented on test data and sample data sets of reinforcements. In the decision tree, each internal node represents a test on an attribute, each branch represents an outcome of the test, and leaf node represents classes or class distribution.

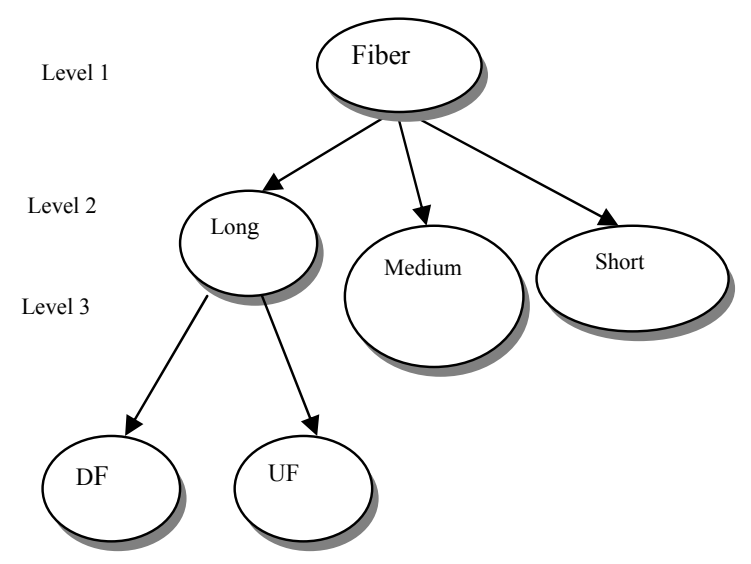

Figure 4: Decision Tree Classifier

The root node at level 1 defined with discrimination function $\mathrm{C}(\mathrm{D}, \mathrm{F})$ on decision class $\mathrm{D}=$ $\left\{\mathrm{d}_{1}, \mathrm{~d}_{2}, \mathrm{~d}_{3}, \mathrm{~d}_{4}\right\}$ containing decision rules and the reinforcement fiber data space $\mathrm{F}_{\mathrm{n}, \mathrm{m}}=\left\{\mathrm{t}_{1}, \mathrm{t}_{2}, \mathrm{t}_{3} . . \mathrm{t}_{\mathrm{n}}\right\}$, containing $n$ tuples and each tuple contains $m$ number of attributes. The discrimination function is linear function $\varphi(f)$ classifies fiber data space into short $(S)$, medium $(M)$ and long $(L)$ fiber classes. Further it discriminates long fiber class into desired (DF) and undesired fiber (UF) classes.

The discrimination function $\varphi(f)$ applied at level 1 for yielding fiber classes L, M and $\mathrm{S}$ is defined with fiber length $L_{f}$ and Modulus of Elasticity $E_{f}$

$$
\varphi(f)=\left\{\begin{array}{lll}
L & \text { if } & \frac{\left(f-E_{f}\right)}{15 l_{c}}>1 \\
M & \text { if } & \frac{l_{c}}{\left(f-E_{f}\right)}<1 \leq \frac{15 l_{c}}{\left(f-E_{f}\right)} \\
S & \text { if } & \left(f-E_{f}\right) \leq l_{c}
\end{array}\right.
$$

Further at the level 2, DTC with function $\varphi(f)$ on fiber class $\mathrm{L}$ discriminates into desired and undesired fiber classes labeled with DFC and UFC respectively. The leaf node DF contains the optimal reinforcement fibers whose length is greater than fifteen times of the critical length, $l_{c}=\frac{\sigma_{f} d}{2 \tau_{c}} m m$ of fiber and $E_{f}$ is greater than $E_{c}$ of polymer composite. The desired fiber (DF) class is being classified by the following function.

$$
\varphi(f)= \begin{cases}D F C & \text { if } \frac{\left(f-L_{f}\right)}{E_{c}}>1 \\ U F C & \text { otherwise }\end{cases}
$$

where $f=W\left(L_{f}+E_{f}\right), W$ is selection quantitative parameter.

\subsection{Cost-Effective Materials Selection Model}

Matching the goals of Knowledge Discovery is the computational part of Data Mining technique. One such technique implemented here is Decision Tree Classifier (DTC) [59] [65][75]. The Decision Tree Classifier is used successfully in many diverse areas such signal processing, pattern recognition, remote sensing, medical diagnosis, expert systems and speech recognition. The most important feature of DTC is its capability to break down a complex decision making process into a collection of simpler decisions. Thus provides a solution which is often easier to interpret.

\subsubsection{Matrix Material Selection Model}

The design cost of composite materials depends on the cost of its constituents materials that meet the design requirements of composite materials. The design cost is minimized with the cost of their constituent materials measured using the units cost per density method.

Therefore, a novel exponential similarity measure model [75] is proposed to measure the homogeneity of material performances on end user requirements. For any two attributes, $\mathrm{x}$ and $\mathrm{y}$, the homogeneity between them is defined as bellow.

$$
d(x, y)=\frac{|x-y|}{1+e^{-|x-y|}}
$$

It satisfies the following principles.

1. $d(x, y) \geq 0 ;$ Distance is a non-negative number. 
A Survey for Data Mining Frame Work

2. $d(x, x)=0$; The distance of an attribute to itself is zero.

3. $d(x, y)=d(y, x)$; The distance is symmetric function

4. $d(x, z)+d(z, y) \geq d(x, y)$; It does not obey triangular inequality.

In general, it can be applied in measuring similarity between two materials say $x_{i}$ and $y_{i}$ having p properties.

$$
d\left(x_{i}, y_{j}\right)=\sum_{k=1}^{p} \frac{\left|x_{i, k}-y_{j, k}\right|}{1+e^{-\left|x_{i, k}-y_{j, k}\right|}}
$$

where $x_{i}=\left\{x_{i, 1}, x_{i, 2}, x_{i, 3}, x_{i, 4}, x_{i, 5}, \ldots \ldots \ldots x_{i, p}\right\}$ and $x_{j}=\left\{y_{i, 1}, y_{i, 2}, y_{i, 3}, y_{i, 4}, y_{i, 5}, \ldots \ldots \ldots y_{i, p}\right\}$

\subsubsection{Cost Computational Model}

It is a core task in KDS for computing appropriate parameters, which have relationship with the output of data mining algorithm [77], for researching for data patterns that constitute core knowledge. For the design requirements of cylindrical shaft given in the chapter 17 of [72], it is required to compute, longitudinal modulus of elasticity, $E_{c}$, Volume , $\mathrm{V}_{\mathrm{c}}$ of composite having intra relationship with mid point deflection $\Delta y$ and a moment of inertia I of cross sectional area with inner and outer diameters $d_{\text {in }}$ and $d_{o}$ respectively.

$$
E_{c}=\frac{4 F L^{3}}{3 \pi \Delta y\left(d_{0}^{4}-d_{\text {in }}^{4}\right)} \mathrm{GPa},
$$

where $\Delta y=\frac{F L^{3}}{48 E I}$ and $I=\frac{\pi}{64}\left(d_{0}^{4}-d_{i n}^{4}\right)$

$$
V_{c}=\frac{\pi L}{4}\left(d_{0}^{4}-d_{i n}^{4}\right) \mathrm{cm}^{3}
$$

The longitudinal modulus of elasticity $E_{c}$ of composite material with long fiber having deflection $\theta$, is derived with composite rule mixture formula [72].

$E_{c}=\operatorname{Cos} \theta\left(E_{m} v_{m}+E_{f} v_{f}\right)$ and $v_{m}+v_{f}=1$.

The volume of composite $V_{c}$, is proportional to the relative volumes $V_{m}$ and $V_{f}$ of Matrix and Fiber respectively. The volumes and masses of these are being computed with composite rule mixture computation.

$$
\left[\begin{array}{ll}
V_{f} & V_{m}
\end{array}\right]=\left[\begin{array}{ll}
v_{f} & v_{m}
\end{array}\right]\left\{\begin{array}{l}
V_{c} \\
V_{c}
\end{array}\right\}
$$

The masses of polymer matrix and fiber vary proportionally with the product of their density and volume respectively.

$\left[\begin{array}{ll}M_{f} & M_{m}\end{array}\right]=\left[\begin{array}{ll}V_{f} & V_{m}\end{array}\right]\left\{\begin{array}{l}\rho_{f} \\ \rho_{m}\end{array}\right\}$

The costs of the Polymer Matrix and fibers are computed with

$\left[\begin{array}{ll}C_{f}^{1} & C_{m}^{1}\end{array}\right]=\left[\begin{array}{lll}M_{f} & M_{m}\end{array}\right]\left\{\begin{array}{l}C_{f} \\ C_{m}\end{array}\right\}$

The cost of composite material is sum of the cost of fiber and the polymer matrix. The algorithm for selecting the cost effective composite materials is described below:

\subsubsection{Algorithms for Fiber Classification and Cost Computation}

\section{Algorithm 1:}

Input : Fiber reinforcement fiber data space $\mathrm{F}$.

Output: A set of selected fibers class DF, each fiber length is greater $151_{c}$

\section{Method:}

Step 1: Create a node N.

Step 2: if samples are all of the same class, $\mathrm{C}$ then return $\mathrm{N}$ as leaf node labeled with the class $\mathrm{C}$;

Step 3: If an attribute list is empty then return $\mathrm{N}$ as the leaf node labeled with class $C$.

[Select a test attribute and determine its class.]

Step 4: Select a test attribute, which is determined heuristically.

Step 5: For each $\mathrm{f}$ in $\mathrm{F}$ satisfying discrimination function $\mathrm{d}_{\mathrm{i}}$, at the $\mathrm{i}^{\text {th }}$ level create a child node for the parent node and labeled it

Step 6: Repeat through step 4 until conditional class $=0$

\section{Algorithm 2:}

Inputs: Table $F_{m x n}\left(L_{f}, C_{f}, \rho_{f}, E_{f}\right)$ contains, length which is greater $151_{c}$, cost, density and tensile modulus of 
Doreswamy

fibers and elastic modulus of composite, $\mathrm{E}_{\mathrm{c}}$, fiber critical length $1_{\mathrm{c}}$.Polymer Matrix table $P_{m x n}\left(C_{m}, \rho_{m}, E_{m}\right)$.

Output: Least cost fiber associated to the given candidate Polymer Matrix.

\section{Method}

Step 1: obtain the output of classifier, $F_{m \times n}(c, d, e)$ of fibers.

Step 2: compute Volume of composite, $\mathrm{V}_{\mathrm{c}}$,

Step 3: Compute for $\mathrm{i}=1$..n do// for a Polymer Matrix and all the fibers.

\section{Begin}

$$
\begin{aligned}
v_{f} & \leftarrow \frac{1}{(\mathrm{E} \mathrm{f}-\mathrm{Em})}\left(\frac{E_{c}}{\operatorname{Cos} \theta}-E_{m}\right) \\
v_{m} & \leftarrow \frac{1}{E m}\left(\frac{E_{c}}{\operatorname{Cos} \theta}-E_{f} v_{f}\right)
\end{aligned}
$$$$
\text { Compute }\left\lfloor V_{f} V_{m}\right\rfloor,\left\lfloor M_{f} M_{m}\right\rfloor,\left[C_{f}^{1} C_{m}^{1}\right] \text {, }
$$

Compute $C_{c}=C_{f}^{1}+C_{m}^{1}$

end

Step 4: Select

$$
\operatorname{Min}\left(C_{c}(i)\right)
$$

Step 5: stop

\subsection{Composite Performance Analysis Model}

It tailors the knowledge discovered by the knowledge discovery models [79] and predicts the general characteristics of the structural composites. This model predicts the mean stiffness of composite laminate in which reinforcement fibers are placed at different orientations in different layers of composite laminate.

\subsubsection{Continuous and Aligned Fibers}

The properties of a composite having its fibers aligned are highly anisotropic, that is independent on the directions in which they are measured. The modulus of elasticity of a continuous and aligned fibrous with very good fibermatrix bond strength or shear yield strength, $\tau_{c}$, in the direction of alignment or longitudinal direction, is proportional to the sum of products of modulus of elasticity and volume of both polymer matrix and fibers reinforced in the polymer matrix composite, whose volume is equal to Area $=$ Length $*$ Breadth * Height composite geometry. A typical arrangement of continuous and aligned fibers along the direction of the applied load is shown in the figure 5.

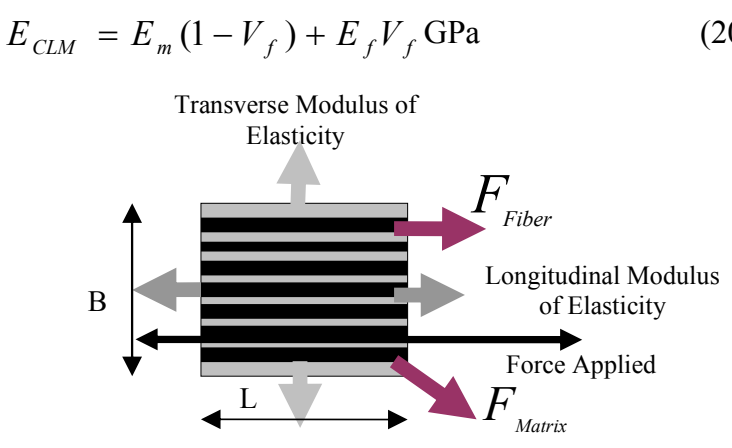

Figure 5: Composite structure having aligned fibers with orientation at $0^{0}$ along the direction of the force applied.

In a fibrous composite with the applied stress aligned perpendicular to the fibers, the stress is transferred to the fibers through the fiber matrix interface and both the fiber and the matrix experience the same stress. This longitudinal modulus of elasticity of composite sets up a vector relationship between modulus of elasticity and volume of both fiber and matrix phases, whose magnitude satisfies the relational boundary of cosine amplitude method [74]. A typical fiber arrangements perpendicular to the direction of force applied is shown in figure 6 and its transverse modulus of elasticity computed by

$$
\frac{1}{E_{C T M}}=\frac{V_{m}}{E_{m}}+\frac{V_{f}}{E_{f}} \quad \mathrm{GPa}
$$

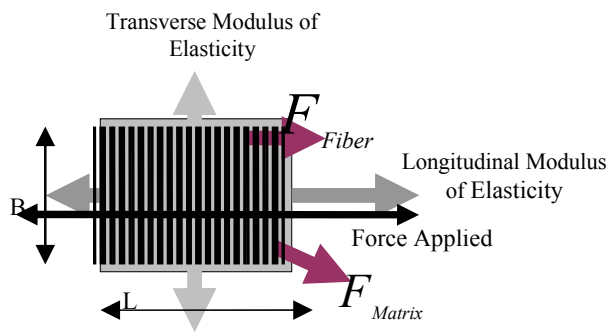

Figure 6: Composite structure having aligned fibers with orientation at $90^{\circ}$ along the direction the force applied.

When the fibers are placed at different angles from $0^{0}-90^{0}$ , the continuous and aligned fibers' modulus of elasticity decreases from the composite strength at $0^{0}$ to the composite strength at $90^{\circ}$. The longitudinal modulus of 
A Survey for Data Mining Frame Work

fibers with orientation $\theta$ along the direction of the applied is measured by

$$
E_{c}=\cos \theta\left(E_{m}\left(1-V_{f}\right)+E_{f} V_{f}\right) \mathrm{GPa}
$$

Typical fiber arrangement at $45^{\circ}$ orientations along the direction of force applied is shown is figure 7.

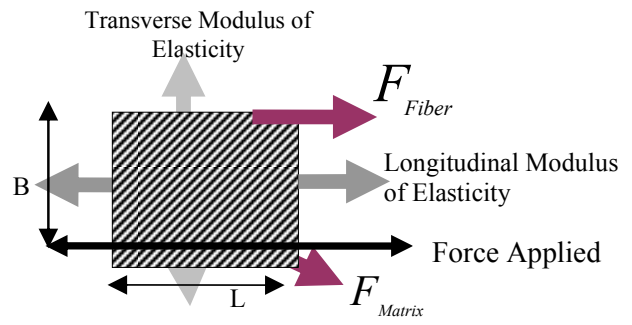

Figure 7: Composite structure having aligned fibers with orientation at $45^{\circ}$ along the direction of the force applied.

The modulus of elasticity of continuous and oriented fiber composite loaded in the transverse direction of the force applied increases with the increase of volume fraction of fibers as the fibers' orientation increases from 0 to 90 degree. This value is computed by the following formula.

$E_{c}=(1-\operatorname{Sin} \theta) \frac{E_{f} E_{m}}{\left(1-V_{f}\right) E_{f}+E_{m} V_{f}} \mathrm{GPa}$

In the composite volume $V_{c}=V_{f}+V_{m}$, such that $V_{f}=V_{c} v_{f}$ and $V_{m}=V_{c} v_{m}$ with $v_{f}+v_{m}=1$.

If the fiber phase volume $V_{f}$ is distribute over $\mathrm{N}$ planes, $\left\{P_{1}, P_{2}, \ldots \ldots P_{N}\right\}$ respectively with fiber volume phases $\left\{V_{f}(i), V_{f}(2), \ldots \ldots . V_{f}(N)\right\}$, which are proportional to fiber volume fractions, $\{v(1), v(2), v(3), v(4), \ldots \ldots . v(N)\}$.

The volume of a single fiber having fiber length $f_{l}$ at $i^{\text {th }}$ plane is $v s f(i)=f_{l} * v(i)$

Number of possible fibers at $i^{\text {th }}$ plane is

$f_{n}(i)=\frac{V_{f}(i)}{v s f(i)}$

The total volume of fiber phase at $i^{\text {th }}$ plane is

$C V_{f}(i)=\operatorname{vsf}(i) * f_{n}(i)=V_{f}(i)$

The plane $P_{i}$ having fiber phase volume, $C V_{f}(i)$ volume fraction $v(i)$ and if all the fibers, $f_{n}(i)$, arranged at $\theta$ degree, the composite longitudinal modulus of elasticity(CLME) and composite transverse modulus of elasticity(CTME) of composite part are computed by the following equations.

$$
\begin{aligned}
& E_{\text {CLME }}\left(P_{i=1 \ldots N}, \theta\right)=\cos \theta\left\lfloor\left(E_{m}\left(V_{c}-V_{f}(i)\right)+E_{f} V_{f}(i)\right)\right\rfloor G P a \\
& E_{\text {CTME }}\left(P_{i=1 \ldots N}, \theta\right)=(1-\operatorname{Sin} \theta)\left[\frac{E_{f} E_{m}}{V_{m} E_{f}+E_{m} V_{f}(i)}\right] \mathrm{GPa}
\end{aligned}
$$

The mean of longitudinal modulus of all the planes $\left\{P_{1}, P_{2}, \ldots \ldots P_{N}\right\}$ in which fibers oriented at $\theta$ degree in a given composite laminate is

$E_{\text {Mean-CLME }}(\theta)=\frac{1}{N} \sum_{i=1}^{N} \cos \theta\left[\left(E_{m}\left(V_{c}-V_{f}(i)\right)+E_{f} V_{f}(i)\right)\right] \mathrm{GPa}$

The mean of transverse longitudinal modulus of all the planes $\left\{P_{1}, P_{2}, \ldots \ldots P_{N}\right\}$ in which fibers oriented at degree $\theta$ is

$E_{\text {Mean }-C T M E}(\theta)=\frac{1}{N} \sum_{i=1}^{N}(1-\operatorname{Sin} \theta)\left[\frac{E_{f} E_{m}}{V_{m} E_{f}+E_{m} V_{f}(i)}\right] \mathrm{GPa}$

In the case of composite consisting of $\mathrm{N}$ planes and each plane, fibers are arranged at $\theta$ (i) degree orientation, Mean LM and Mean TM are computed by

$E_{\text {Mean }-C L M E}=\frac{1}{N} \sum_{i=1}^{N} \cos \theta(i)\left[\left(E_{m}\left(V_{c}-V_{f}(i)\right)+E_{f} V_{f}(i)\right)\right] \mathrm{Gpa}$

$E_{\text {Mean }- \text { CTME }}=\frac{1}{N} \sum_{i=1}^{N}(1-\operatorname{Sin} \theta(i))\left[\frac{E_{f} E_{m}}{V_{m} E_{f}+E_{m} V_{f}(i)}\right] \mathrm{Gpa}$

A composite consisting of different planes and in each plane fibers oriented at $\theta$ degree is shown in figure 8 .

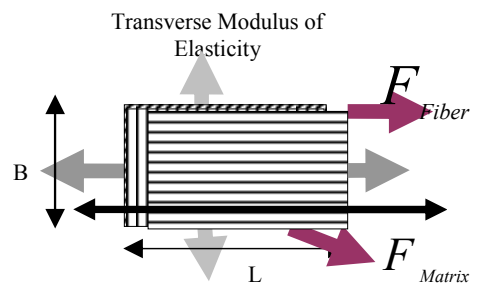

Figure 8: Composite structure having aligned fibers at unique orientation in each plane of the composite.

\section{Data Evaluation And Representation}

Data evaluation and representation is a vital component of data mining and knowledge discovery processes to visualize the knowledge and validating the knowledge of interestingness. The materials in data sets are classified into different materials classes such as Metal, Ceramic and Polymer by the back propagation neural network classification model that consists of 25 inputs, 7 hidden 
Doreswamy

and 3 output neurons. The learning curves with varying learning rates with constant momentum are shown in the figure 9. The classification model is efficient in classifying 5000 matrix materials with mean classification accuracy $99.35 \%$ and mean classification error accuracy $0.00690 \%$, less than $1 \%$.

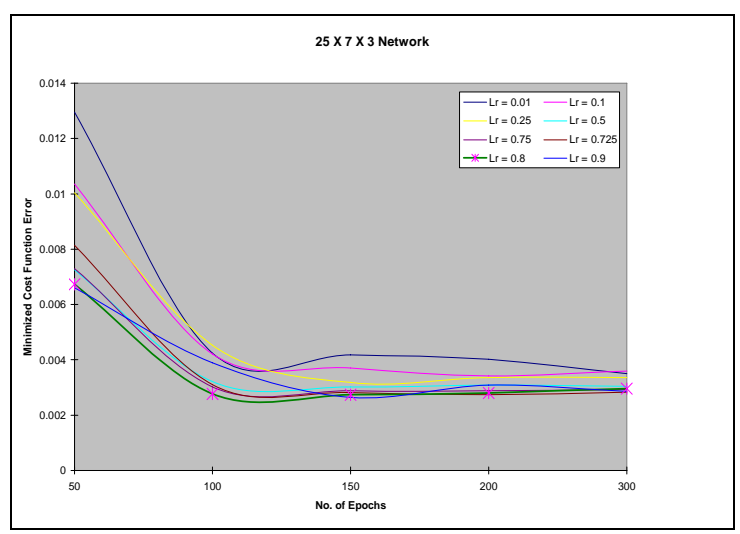

Figure 9: Network structure $25 \times 7 \times 3$ with learning curves for varying learning rates and fixed momentum constant.

Material selection model selects polymer matrix: polycarbonate material that match the input design requirements, from the polymer class. Classified long fiber class materials are considered for computing cost of composite materials. Cost of the polymer matrix composite \{Polycarbonate + Aramid that matches the end user requirements is computed with a set of long finer and is depicted in the figure 10 .

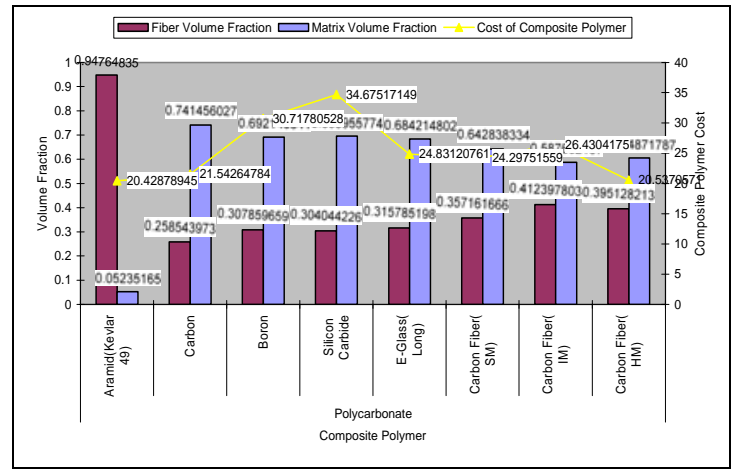

Figure 10: Shows the volume fractions of both Polymer Matrix and Reinforcement fibers and the cost of polymer composites: polycarbonate with different reinforcement fibers.
The knowledge extracted from both the polymer matrix and fiber reinforcement selection tools are tailored for the analysis of the fiber performance in composite using composite properties. The composite performance analaysis model analyzes the longitudinal modulus and transverse modulus of the composite material. Fibers phase with varying volume fraction and diameter are placed at different orientations in different planes. The longitudinal modules and the transverse modulus of the composite material having the geometrical features specified in the table 2 and having different volume fractions of the fibers placed in it at orientations $0,15,30$, $45,60,75$, and 90 degree at different plans, respectively decreases gradually to zero and increases to one that is shown in the figure 11.

Table 2: Physical and geometrical properties of a composite part

\begin{tabular}{|c|l|l|}
\hline 1 & Volume & $\mathrm{V}=7 * 250 \mathrm{Cm}$ \\
\hline 2 & Length & $\mathrm{L}=25 \mathrm{Cm}$ \\
\hline 3 & Breadth & $\mathrm{B}=10 \mathrm{Cm}$ \\
\hline 4 & $\begin{array}{l}\text { Height/Number of layers } \\
\text { in composite }\end{array}$ & $\mathrm{N}=7 \mathrm{No}$. \\
\hline 5 & Size of each layer & $25 * 10 \mathrm{~cm}^{2}$ \\
\hline 6 & Orientation of fibers & $\theta$ degree \\
\hline
\end{tabular}

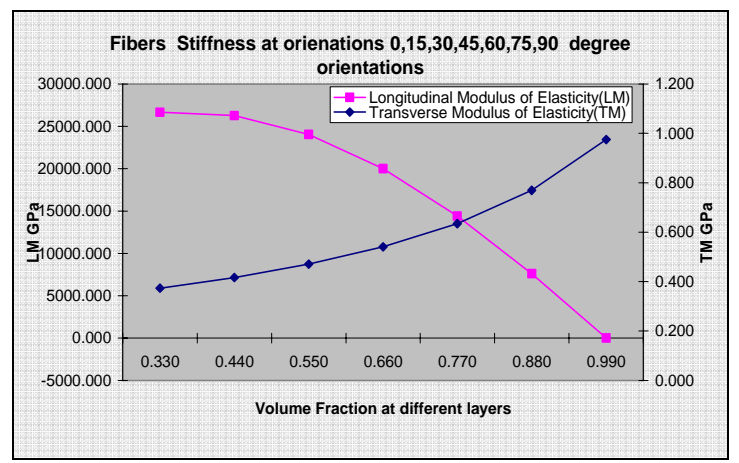

Figure 11: Longitudinal and Transverse modulus of elasticity of composite with fibers orientations at $0,15,30,45,60,75$, and 90 degrees.

The mean stiffness of composite materials with varying fibers features can be maximized and can guide for a design engineer to make strategic decision making that satisfies the end user requirements. 


\section{Conclusion and Future Work}

In this paper an exhaustive literature survey for data mining frame work for engineering materials design applications has been made. An object-Oriented data model is proposed to organize materials data using objects and classes and their enhanced features to simplify searching and retrieval operations. Machine learning technique such as decision tree classifier and AI neural network classifier are proposed for materials classification, where as new similarity measuring technique is implemented for materials selection. The proposed data mining frame work simulates the manual computational operations as done by a composite design engineer and performs the voluminous of computational steps required for maximizing the structural mechanical performance of composite material. Introduction of data mining system to engineering materials design applications improves computational accuracies and design throughput of new engineering materials. This model can assist the design engineers in the selection process of cost effective materials and can guide the designer in maximizing the mechanical stiffness of a composite materials having fiber phases varying volume fractions at different orientations. Further a prototype data mining model is proposed to implement under Soft computing frame work to measure the mechanical performance of composite materials under different conditions.

\section{Acknowledgement}

The author would like to thank the all the anonymous referees for their kind review comments for improving the quality this paper.

\section{References}

[1]. Stephen L. Rosen,(January 1993) Fundamental Principles of Polymeric Materials (Society of Plastics Engineers Monographs).

[2]. Vishu Shah.(October 23,1998) Handbook of Plastics Testing Technology (Society of Plastics Engineers Monographs).

[3]. Bernhardt, E.C. (1959) Processing of Thermoplastic Materials, Reinhold, New York.
[4]. McKelvey, J.M. (1962) Polymer Processing, Wiley, New York.

[5]. Klein, J. and Marshall, D.I. (1968) Computer Programs for Plastics Engineers, Reinhold, New York.

[6]. Tadmor, Z. and Klein. I.(1970) Engineering Principles of Plasticating Extrusion, Wiley-Interscience, New York.

[7]. EXTRUD, SPR, Somerset, NJ, U.S.A.

[8]. MOLDFLOW, Kilsyth, Victoria, Australia.

[9]. C-MOLD, AC TECH, Ithaca, NY, U.S.A.

[10]. Manzione, L.T. (1987) Applications of Computer-Aided Engineering in Injection Molding, Hanser, Munich.

[11]. FIDAP, F.D.I, Evanston, IL, U.S.A. (now owned by FLUENT INC.).

[12]. POLYFLOW, Louvain-La-Neuve, Belgium. (now owned by FLUENT, INC.).

[13]. NEKTON, Creare.x, Hanover, NH, U.S.A. (now owned by FLUENT, INC.).

[14]. POLYCAD ${ }^{\circledR}, \quad$ CAPPA-D, Chemical Engineering, McMaster University, Hamilton, Ontario, Canada. (now owned by Polydynamics, Inc., Hamilton, Ontario, Canada)

[15]. Isayev, A.I.(1987) Injection and Compression Molding Fundamentals, Marcel Dekker, Inc., New York.

[16]. Karagiannis, A., Hrymak, A.N. and Vlachopoulos, J. (1988) AIChE J., 34, 2088.

[17]. Karagiannis, A., Hrymak, A.N. and Vlachopoulos, J. (1989), Rheol. Acta, 28, 121.

[18]. Tucker, C.L.(1989) Fundamentals of Computer Modeling for Polymer Processing, Hanser Publishers, New York.

[19]. SSD, Single Screw Designer (formerly PASS), P.P.I., Stevens Institute of Technology, Hoboken, NJ, U.S.A.

[20]. REX, Institute for Kunststoff technologies, University of Paderborn, Paderborn, Germany,

[21]. CHEMEXTRUD, CEMEF, École des Mines, Sophia Antipolis, Valbonne, France.

[22]. EXTRUCAD, Vlachopoulos, J., Silvi, N. and Vlcek, J. (1992) Japan Plastic., 43(5), 21. 
Doreswamy

[23]. MacGregor, A., Vlachopoulos, J. and Vlcek, J. (1996) SPE ANTEC Proceedings, Indianapolis.

[24]. Martelli, F.G. (1983) Twin-Screw Extruders: A Basic Understanding, Van Nostrand Reinhold, New York.

[25]. White, J.L.( 1990) Twin Screw Extrusion, Hanser Publishers, New York.

[26]. AKRO-CO TWIN SCREW®, University of Akron, Akron, OH, U.S.A.

[27]. POLYDYNAMICS INC., Hamilton, Ontario, Canada. Web site: www.polydynamics.com.

[28]. Garcia-Rejon, A. (1995) Advances in Blow Molding Process Optimization, RAPRA Rev. Reports, 7(10).

[29]. Pro/ENGINEER, Parametric Technology Corp., Waltham, Mass., USA.

[30]. HYPERMESH, Altair Computing, Troy, MI, USA.

[31]. Crowe, E.R. and Vassiliadis, C.A.( 1995), Chemical Engineering Program,USA.

[32]. POLYEXPERT S.A., Athens, Greece.

[33]. O'Brien, K.T.( 1992) Computer Modeling for Extrusion and Other Continuous Polymer Processes, Hanser Publishers, New York,

[34]. Baird and D.G.Collias.(1995) Polymer Processing,Butterworth-Heinemann, Boston.

[35]. J.Lachopoulos.(Februaru 1998) Recent Progress And Future Challenges In Computer-Aided Polymer Processing Analysis And Design, Centre for Advanced Polymer Processing and Design(CAPPA-D), Presented at the ATV-Semapp Meeting, Funen, Odense, Denmark.

[36]. Rayson, R.T.(1985) A Review of Expert System Principles and Their Role in Manufacturing Systems, Robatica, Vol.3, pp.279-287.

[37]. Nielsen, E. H, Dixon, J,R., and Simmons, M K. (1986) GERES: A Knowledge Based Material Selection Program For Injection Molded Resigns, Computers in Engineering American Society of Mechanical Engineers, New Yark, Vol.1,pp. 255-261.

[38]. Megnes, G.,Hovelanns N., and Baur E.( 1987.) Intelligent Computer Program For Plastic Processing, Expert Systems In production Engineering, Lecturer Notes in Engineering, Vol.29, pp.1-19.
[39]. D.C. Brown, and B. Chandrasekaran.(1989) Design Problem solving: facts Structures and Control Strategies, Morgan Kaufmann publication, USA.

[40]. Nitsche, H. Kern, and J. Janczak.( 1990) Composites' Design Based on Expert Knowledge, Proc. Am. Soc. for Composites Fifth Technical Conf., pp.382-390, Technomic Publishing Company, Lancaster.

[41]. Kamel, and J. Sticklen.(1990) An Artificial IntelligenceBased Design Tool for Thin Film Composite Materials, Proceedings American Society for Composites Fifth Technical Conf., pp. 375-381, Technomic Publishing Copany, USA.

[42]. Jon Sticklen, Ahmed Kamel, Martin C. Hawley, John Delong J.(1992) An Artificial Intelligence-Based Design Tool for Thin Film Composite Materials, Applied Artificial Intelligence, Vol. 6, No. 6, pp.303-313.

[43]. Jon Sticklen, Ahmed Kamel, Martin C. Hawley, Valerie Adegbite(1992) Fabricating Composite Materials: A Comprehensive Problem-Solving Architecture Based on Generic Tasks, IEEE Expert, Vol. 7, No. 2, pp. 43-53.

[44]. J. McDowell.( 1993) Domain Structure and Inference Strategies in Knowledge-Based Design of Polymer Composites, SAE Technical Paper \#930167, Society of Automotive Engineers, Detroit.

[45]. Lenz, T., J. K. McDowell, B. Moy, J. Sticklen, and M. Hawley(1994) Intelligent Decision Support for Polymer Composite Material Design in an Integrated Design Environment, Proc. Am. Soc. for Composites Ninth Technical Conference,, pp. 685-691, Technomic Publishing Company.

[46]. Moy, B., McDowell, J. K., Lenz, T., Sticklen, J., \& Hawley, M. C. (1994). Expansion of an Intelligent Decision Support System for Process Selection and Process Design in Polymer Composites. In 26th International SAMPE Technical Conference, pp. 162-171, Atlanta, Georgia.

[47]. Timothy Lenz (HP) and James K. McDowell (HP) and Ahmed Kamel (HP) and Jon Sticklen (HP) and Martin C. Hawley (HP).(1995) A Decision Support Architecture for Polymer Composites Design: Implementations and Evolution, Proceedings of the Eleventh Conference on Artificial Intelligence for Applications, pp. 102-108, IEEE Computer Society Press. 
A Survey for Data Mining Frame Work

[48]. Timothy J. Lenz, James K. McDowell, Martin C. Hawley, Ahmed Kamel, Jon Sticklen,(1996) The Evolution of a Decision Support Architecture for Polymer Composites Design, IEEE Expert: Intelligent Systems and Their Applications, Vol. 11, No. 5, pp. 77-83.

[49]. James K. Mcdowell, Ahmed M. Kamel, Jon Sticklen, Martin C. Hawley. (1996) Integrating Material/Part/Process Design for Polymer Composites: A Knowledge-Based Problem-Solving Approach, Journal of Thermoplastic Composite Materials, Vol. 9, No. 3, pp. 218-238.

[50]. T. J. Lenz, M. C. Hawley, and Jon. Sticklen.(1998) Virtual Prototyping in Polymer Composites", Journal of Thermoplastic Composite Materials, Vol.11, pp.394-416.

[51]. Lenz, T., McDowell, J. K., Moy, B., Sticklen, J., \& Hawley, M. C.(1994) Intelligent Decision Support for Polymer Composite Material Design in an Integrated Design Environment, In American Society of Composites 9th Technical Conference on Composite Materials, pp. 685-691, Newark, Delaware.

[52]. Moy, B., McDowell, J. K., Lenz, T. J., Sticklen, J., \& Hawley, M. C.(1995) Integrated Design and Agile Manufacturing in Polymer Composites. The Role of Intelligent Decision Support Systems, In Intelligent Manufacturing Systems Workshop, 14th International Joint Conference on Artificial Intelligence, IJCAI 95, Montreal.

[53]. Michaeli, W., and A. Biswas.( 1994) Knowledge- and Case-based Computer Support for the Design of Composite Parts, Proceedings of the ANTEC '94, pp.2347-2350, San Francisco.

[54]. Edwards, K. L., P. M. Sargent, \& D. O. Ige.( 1994) Designing with Composite Materials - an Expert System Approach, Proceedings the American Society of Mechanical Engineers 2nd Biennial European Conference on Engineering Systems Design \& Analysis, London.

[55]. Netten, B. D., R. A. Vingerhoeds, \& H. Kippelaar.( 1995) Expert Assisted Conceptual Design: An Application to Fibre Reinforced Composite Panels, In R. M.Oxman, M. F. T. Bax, \& H. H. Achten (Eds.), Design Research in the Netherlands, pp. 125-139, Eindhoven: Faculty of
Architecture, Planning, and Building Science. Eindhoven University of Technology.

[56]. Krzysztof J Cios, Georgr Y. Baaklini and Alex Vary.( 1993) Soft Computing in Design and Manufacturing of Advanced Materials, Technical report at NASA. Prepared and submitted for the American Society of Mechanical Engineers cosponsored by the IGTI and ASME, Cincinnati.

[57]. Sushmita Mitra, Shankar K.Pal and Pabtra.(2002) Data Mining in Soft Computing Frame: A Survey, IEEE Tran sanctions on Neural Networks, Vol.13, No.1. pp.no.3-14.

[58]. Jiawei Han, Russ B. Altaman, Vipin Kumar, Heikki Mannila, and Daryl Pregibon. (2002) Emerging Scientific applications in Data Mining, Communications of $A C M$, Vol.45.No.8.

[59]. Michael Goebel and Le Gruenwald.(1999) A Survey of Data Mining and Knowledge Discovery Software Tools, $A C M$ SIGKDD, Vol.1. Issues 1, pp.20-32.

[60]. A Abrham, B.D. Baets, M.Koppen, and B.Nickolay.(2006) "Applied Soft Computing Technologies: The Challenges of Complexity" Springer publication.

[61]. Simon, H.(1969) The Sciences of the Artificial, The M.I.T. Press, Cambridge, USA.

[62]. Xindog Wu. (2004) Data Mining: An Artificial Intelligence Perspective, IEEE Computational Intelligence Bulletin, Vol.4.No.2.

[63]. Ming-Syan Chen, Jiawei Han, and Philip Yu.(1996) Data Mining: An Overview from a Database Perspective, IEEE Transactions on Knowledge and Data Engineering, Vol.8, No.6, pp.866-883.

[64]. John Elder IV and Daryl Pregibon. (1996) A Statistical Perspective on Knowledge Discovery in Databases, in Advances in Knowledge Discovery and Data Mining, Usama M. Fayyad, Gregory Piatetsky-Shapiro, Padhraic Smyth, and Ramasamy Uthurusamy (Eds.), AAAI Press, pp. 83-113.

[65]. Jiawei Han and Micheline Kamber, Data Mining (2000) Concepts and Techniques, Morgan Kaufmann publication,USA. 
Doreswamy

[66]. Stuart Russell and Peter Norvig. (2003) Artificial Intelligence: A Modern Approach, Second Edition, Prentice-Hall.

[67]. X. Wu.(1995) Knowledge Acquisition from Databases, Ablex Publishing Corp., U.S.A.

[68]. S Zhang, C Zhang, and X Wu.(2004) Knowledge Discovery in Multiple Databases, Springer-Verlag.

[69]. Zhi-Hua Zhou. (2003) Three Perspectives of Data Mining, Artificial Intelligence, Vol.143, No.1, pp. 139146.

[70]. Christopher C. Fischer,Kevin J. Tibbetts, Dane Morgan and Gerbrand Ceder (2006) Predicting Crystal Structure By Merging Data Mining With Quantum Mechanics, Nature Materials, Vol.5, No.8, pp.641-646.

[71]. Pedro Domingous, toward knowledge-Rich Data Mining (2007) Data Mining and Knowledge Discovery, vol.15, pp.21-28.

[72]. William D Callister, Jr. (1997) Materials Science and Engineering an introduction, forth edition, John Wiley \& Sons Incorporation.

[73]. Ussama Fayyad, Gregarory Piatetsky-Shapiro and Padharic Smyth ( 1996) From Data Mining to Knowledge Discovery in Databases, American Association for Artificial Intelligence, pp.37-54.

[74]. Timothy J. Ross.(1997) Fuzzy Logic with Engineering Applications, McGraw-Hill Inc.

[75]. Doreswamy, S. C. Sharma, (June 2006) An Expert Decision Support System for Engineering Materials Selections And Their Performance Classifications on Design Parameters, International Journal of Computing and Applications (ICJA), Vol.1, No.1,pp.17-34..

[76]. Doreswamy, (August 2007) Engineering Materials Classification Model- A Neural Network Application, IJDCDIS A Supplement, Advances in Neural Networks. Vol.14 (S1), 591-595, 2007.

[77]. Doreswamy, S. C Sharma and M Krishna, Knowledge Discovery System for Cost-Effective Composite Polymer Selection-Data Mining Approach, Proceedings of 12th International Conference on Management of Data COMAD 2005b, pp.no. 185-190.
[78]. Doreswamy, S. C. Sharma, M.Krishna, and H. Narsimhamoorthy, (2006) Data Mining Application In Knowledge Extraction Of Polymer And Reinforcement Clustering, Proceedings of International Conference on Systemics, Cybernetics And Informatics, ICSCI-2006, Vol. 1, pp.no.562-566.

[79]. Doreswamy, (2007) Polymer Matrix Composite Performance Analysis Knowledge Engineering Model, proceedings of International Conference on Systemics, Cybernetics and Informatics, ICSCI-2007, Vol. No.1, pp.305-315.

[80]. Doreswamy, (2008) Machine Learning Classification and Selection Model for Engineering Materials Design Applications, Proceedings of International Conference on Systemics, Cybernetics and Informatics, ICSCI-2008, Vol.1, pp.no.739-743. 